In: Gesundheitsreform 2007. Nach der Reform ist vor der Reform. Hrsg.: Schroeder (u.a.). Wiesbaden: Verlag für Sozialwissenschaften. S. 112-117

\title{
Zur Verfasstheit gesetzlicher und privater Krankenversicherungsunternehmen nach der Gesundheitsreform 2007
}

\author{
Andreas Hälein
}

\section{Einleitung}

Wenn Robert Paquet in seinem nachfolgenden Beitrag wiederholt die unternehmerische Qualität der Gesetzlichen Krankenkassen nach dem GKV-Wettbewerbsstärkungsgesetz (GKV-WSG) betont, spricht er damit eine sich seit langem abzeichnende Entwicklung an, die die Reform erneut ein deutliches Stück vorangetrieben hat: Die Krankenkassen werden unternehmerisch, unternehmensähnlich, und verstehen sich offenbar auch so - einerseits. Anderseits gibt es mit der Stärkung der Stellung des Staates im Krankenversicherungswesen eine scheinbar gegenläufige Entwicklung. Für die Private Krankenversicherung zeigt Timm Genett, dass das Reformgesetz deren Unternehmen Elemente von Zwangsversicherung und Solidarausgleich verordnet, wenn auch nicht so weitgehend, wie ursprünglich geplant.

Die angedeuteten Entwicklungen sollen hier nicht in allen rechtlichen Einzelheiten nachgezeichnet werden. Das rechtswissenschaftliche Interesse gilt vielmehr den wechselseitigen Bezügen zwischen dem neuen Gesetzesrecht und dem rechtlichen Rahmen, in den es eingebettet ist: dem deutschen Verfassungs- und dem europäischen Wirtschaftsrecht.

\section{Die Krankenkassen im Staatsgefüge}

Krankenkassen sind Selbstverwaltungskörperschaften; sie zählen zur mittelbaren Staatsverwaltung. Über den Krankenkassen hat sich im Lauf einer jahrzehntelangen Entwicklung eine komplexe Verbandsstruktur aufgebaut. Da gibt es Landesund vor allem Bundesverbände und andere Spitzenverbände der verschiedenen 
Kassenarten. Neben diesem Verbandssystem existierten Verbandssysteme der Leistungserbringer, insbesondere der Ärzteschaft und im Krankenhauswesen. Die Verbandsstrukturen von Kassen und medizinischen Dienstleistern sind in Form der sogenannten "gemeinsamen Selbstverwaltung" zusammengekoppelt; diese erfüllt zahlreiche Aufgaben der Konkretisierung gesetzlicher Vorgaben. Den Schlussstein dieser Architektur bildet der Gemeinsame Bundesausschuss, in dem Vertreter aus den verschiedenen Säulen der gemeinsamen. Selbstverwaltung über grundlegende Fragen der medizinischen Versorgung entscheiden. Der Umfang der Entscheidungsbefugnisse, die der Gesetzgeber hier aus der Hand gegeben hat, ist bemerkenswert und seit längerem Gegenstand verfassungsrechtlicher Kontroversen. Die mächtigen Verbandsspitzen haben sich im Übrigen zu lautstarken Akteuren im gesundheitspolitischen Diskurs entwickelt, die ihre Interessen selbstbewusst zu artikulieren verstehen.

Die Politik hat sich nun bemüht, den eigenen Einfluss, den Einfluss des Staates deutlich zu stärken und zugleich die bisherigen Kassenverbände partiell zu entmachten. So soll mit dem neuen "Spitzenverband Bund" die Kooperation der verschiedenen Kassenarten auf Bundesebene "deutlich gestrafft" und „Handlungsblockaden vermieden werden" ( s. BT-Drs. 16/3100, S. 90). Änderungen an der Konstruktion des Gemeinsamen Bundesausschusses verfolgen das Ziel, die Akteure von "den Interessenlagen der ,Bänke” zu lösen und „sachbezogenere und weniger interessengeleitete Entscheidungen" zu unterstützen (ebd. S. 179).

$\mathrm{Zu}$ einer Stärkung des staatlichen Einflusses führt ferner auch die Errichtung des Gesundheitsfonds und die Verlagerung der Beitragsfestsetzung auf die Bundesregierung ab dem 1.1.2009. In die gleiche Richtung weist die Erhöhung des Bundeszuschusses zur Gesetzlichen Krankenversicherung, der nach und nach auf 14 Milliarden Euro anwachsen wird. Die Beteiligung des Bundes an der Finanzierung der Gesetzlichen Krankenversicherung zieht Kontrollrechte des Bundesrechnungshofs nach sich ( $\$ 274$ Abs. 5 n.F.).

Die Reformelemente, die auf eine Stärkung des staatlichen Einflusses abzielen, sind aus verfassungsrechtlicher Sicht von hohem Interesse. Insbesondere erscheint möglicherweise die vom Bundesverfassungsgericht bisher ausdrücklich offen gelassene Frage in einem neuen Licht, ob die untergesetzlichen gesundheitspolitischen Regelungskompetenzen der gemeinsamen Selbstverwaltung, in erster Linie des Gemeinsamen Bundesausschusses, in einem demokratischen Staatswesen überhaupt akzeptiert werden können. Diese Frage wird etwa von dem Verfassungsrechtler Kingreen verneint: Auch oder erst recht nach der Reform von 2007 sieht er den Gemeinsamen Bundesausschuss "endgültig im Niemandsland zwischen unmittelbarer und mittelbarer Staatsverwaltung ver- 
schwinden" (s. Kingreen 2007a). Man kann dies freilich auch anders sehen: Wächst der Einfluss der Ministerin oder des Ministers im Zusammenhang mit Regelungsbefugnissen des Kassensystems, lassen sich Entscheidungen einem persönlich verantwortlichen und demokratisch legitimierten Amtsträger zurechnen, der - anders als ein Verbandsvertreter - die nächste Bundestagswahl fürchten muss (vg1. Hänlein 2007).

\section{Unternehmen der Krankenversicherung}

Die Gesetzlichen Krankenkassen werden mehr und mehr zu Unternehmen. Diese Entwicklung, die das Gesundheitsstrukturgesetz 1992 angestoßen hatte, wird durch das aktuelle Reformgesetz erneut vorangetrieben. So können Krankenkassen jetzt leichter fusionieren, weil Zusammenschlüsse von Krankenkassen nun nicht mehr nur innerhalb einer Kassenart möglich sind. Das Gesetz fasst für die Zukunft ausdrücklich die Insolvenzfähigkeit der Krankenkassen ins Auge. Den Kassen werden mit den Instrumenten des Selbstbehalts und der Beitragsrückerstattung (genannt „Prämienzahlungen") Geschäftsmodelle ermöglicht, wie sie aus der Privaten Krankenversicherung bekannt sind und die nach traditionellem Verständnis in ein solidaritätsgeprägtes System nicht hineinpassen ${ }^{1}$. Und im Verhältnis zu den Leistungserbringern, insbesondere zu den Ärzten, genießen die Krankenkassen ebenfalls zunehmend unternehmerische Freiheiten. So können sie zur Sicherstellung der hausarztzentrierten Versorgung selektiv Verträge mit Ärzten ihrer Wahl abschließen.

Je mehr die Krankenkassen sich von Behörden zum Unternehmen wandeln, desto schärfer stellt sich die Frage, ob für sie auch die Regularien gelten, die auch sonst von Unternehmen am Markt zu beachten sind. Brisant ist insbesondere das Problem, ob das Marktgebaren der Krankenkassen wettbewerbsrechtlichen Regeln unterliegt. Haben sie das UWG zu beachten? Wie verhält es sich mit den Regeln des nationalen oder des europäischen Kartellrechts?

In den 1990er Jahren hatten die Kartellgerichte dazu tendiert, die Krankenkassen dem Kartellrecht zu unterwerfen (vgl Kruse/ Hänlein 2000). Der Gesetzgeber hat dann versucht, dem einen Riegel vorzuschieben: Seit dem 1.1.2000 durften auf die Beziehungen der Krankenkassen zu den Leistungserbringern ausschließlich die Vorschriften des SSGB V angewendet werden (\$ 69 SGB V i.d.F.

${ }^{1} \mathrm{Zu}$ einem früheren Versuch, derartige Instrumente einzuführen, siehe: Kommentierung der $\$ 53$ u. 54 a.F (Kruse 1999). 
des GKV-Gesundheitsreformgesetzes 2000 vom 22.12.1999, BGBL. I, S. 2626). Zugleich wurde damals den Kartellgerichten die Zuständigkeit fiir dieses Feld entzogen. Später hat auch der Europäische Gerichtshof (EuGH) in der Tendenz ähnlich entschieden. In seinem "Festbetragsurteil” hat er die Auffassung vertreten, die Ortskrankenkassen seien von vornherein nicht als Unternehmen im Sinne des europäischen Kartellrechts anzusehen, weil sie eine rein soziale Aufgabe wahrnähmen, die auf dem Grundsatz der Solidarität beruhe und ohne Gewinnerzielungsabsicht ausgeübt werde (EuGH, Urteil v. 16.3.2004 - Rs. C-264, 306, 354 und 355/01 - Slg. I 2004, S. 2493).

Vor diesem Hintergrund ist es nachgerade spektakulär, dass der Reformgesetzgeber nunmehr ausdrücklich Teile des nationalen Kartellrechts für anwendbar erklärt hat ${ }^{2}$. Es ließ sich schlicht nicht länger durchhaiten, den Krankenkassen. etwa gegenüber den Apotheken zu erlauben, ihre Nachfragemacht in einer Weise auszuspielen, wie man es bei privaten Unternehmen niemals hinnehmen würde. Vermutlich ist es nur eine Frage der Zeit, bis der EuGH aus dem Wandel der deutschen Krankenkassen den Schluss ziehen wird, sie seien auch im Sinne des europäischen Kartellrechts doch als Unternehmen zu behandeln ${ }^{3}$ Und es ist nur konsequent, dass sich das Bundeskartellamt zur Fusionskontrolle nach dem GWB befugt sieht, wenn Krankenkassen fusionieren (vg1. Gaßsner/ Ahrens 2007).

Während die Krankenkassen nach und nach immer unternehmensähnlicher werden, lässt sich im Bereich der Privaten Krankenversicherung eine gegenläufige Entwicklung verzeichnen: Hier hat das Reformgesetz Elemente einer solidarischen Versicherung eingeführt, insbesondere in Form des Basistarifs, den die Versicherer von Gesetzes wegen gewissen Personengruppen anzubieten haben. Zugleich verpflichtet das Gesetz die Versicherungsuntemehmen auf einen Ausgleich der Versicherungsrisiken im Basistarif. Über die rechtlichen Konsequenzen der verordneten Konvergenz gesetzlicher und privater Krankenversicherer wird noch näher nachzudenken sein. Was folgt daraus, dass - durch die immense Erhöhung des Bundeszuschusses in der Gesetzlichen Krankenversicherung - die Solidarität innerhalb des GKV-Systems abgeschwächt wird, während zugleich

\footnotetext{
${ }^{2} \S 69$ Abs. 1 S. 2 HS 1 SGB V n.F.: „Die $\$ \$ 19$ bis 21 des Gesetzes gegen Wettbewerbsbeschränkungen gelten entsprechend"; § 19 GWB: Verbot missbräuchlicher Ausnutzung einer marktbeherrschenden Stellung; $\$ 20$ GWB: Diskriminierungsverbot und Verbot unbilliger Behinderung durch marktbeherrschende Unternehmen; \& 21 GWB: Boykottverbot und Verbot sonstigen wettbewerbsbeschränkenden Verhaltens; zu § 69 SGB V n.F. auch Möschel 2007

${ }^{3}$ So auch Kingreen: "Wer Wettbewerb sät, wird eben irgendwann Wettbewerbsrecht ernten"(s. Kingreen 2007b; ähnlich Schlegel 2006).
} 
die Private Krankenversicherung intern auf Solidarität verpflichtet wird? Diese Entwicklung wird den Diskussionen über den Wettbewerb in der Krankenversicherung ganz neue Dimensionen verleihen. Ging es bisher meist um die Frage, ob Krankenkassen als Nachfrager medizinischer Leistungen Wettbewerbsregeln zu beachten haben, wird künftig der Wettbewerb zwischen den Anbietern des Produkts "Sicherung im Krankheitsfall" in den Blick geraten, die Frage also, ob und in welchem Umfang es Wettbewerb zwischen gesetzlichen und privaten Versicherern geben kann, soll oder muss. Genau gesehen hat die Diskussion schon begonnen: Für Kingreen folgt aus der Entsolidarisierung innerhalb des GKVSystems infolge des hohen Bundeszuschusses, dass die Rechtfertigung des Monopols der Krankenkassen für den Schutz der Pflichtversicherten aus europarechtlicher Sicht brüchig wird, eine Öffnung des Systems für private Anbieter würde diesen dann auch Zugang zum steuerfinanzierten Solidarausgleich eröffnen, von dem sie gegenwärtig noch ausgeschlossen sind (vgl. Kingreen 2007b).

\section{Fazit}

Festzuhalten bleibt: Das GKV-Wettbewerbsstärkungsgesetz stärkt zunächst einmal den Staat, eine Entwicklung, die auch von verfassungsrechtlicher Relevanz ist, weil sie zu transparenteren Entscheidungsstrukturen führen kann. Den Krankenkassen verschafft das Reformgesetz weitere unternehmerische Freiheiten, so dass die Frage nach der Anwendung wirtschaftsrechtlicher Grundsätze aktueller denn je geworden ist. Partielle Entsolidarisierung in der Gesetzlichen Krankenversicherung und solidarische Strukturelemente in der Privaten Krankenversicherung könnten überdies das Versicherungsmonopol der Gesetzlichen Krankenversicherung auf den Prüfstand stellen. Die aus-der Frühzeit der Gesetzlichen Krankenversicherung bekannte Debatte zum Thema "Zwangskasse oder Kassenzwang" (s. Tennstedt/ Lohmann) könnte so in neuem Gewand eine Neuauflage erleben.

\section{Literatur}

Gaßner, Maximilian/ Ahrens, Hans Jürgen (2007): Anwendbarkeit der Regeln der Fusionskontrolle des GWB bei der Vereinigung gesetzlicher Krankenkassen, SGb, S. $528 \mathrm{ff}$.

Hänlein, Andreas (2007): Anmerkung zum Urteil des BSG v 4.4.2006 - B 1 KR 12/04 R - (DRibose), SGb, S. 169, $172 \mathrm{f}$. 
Hänlein, Andreas/ Kruse Jürgen (2000): Einflüsse des Europäischen Wettbewerbsrechts auf die Leistungserbringung in der gesetzlichen Krankenversicherung, NZS 2000, S. 165 ff.

Kingreen, Thorsten (2007a): Legitimation und Partizipation im Gesundheitswesen, NZS, S. $113,120$.

Kingreen, Thorsten (2007b): Soziale und private Krankenversicherung: Gemeinschaftsrechtliche Implikationen eines Annäherungsprozesses, ZESAR 2007, S. 139, 149.

Kruse, Jürgen (1999) In: Kruse, J./ Hänlein, A.. LPK-SGB V, 1. Aufl., 1999.

Möschel, Wernhard (2007): Gesetzliche Krankenversicherung und das Kartellrecht, JZ, S. $601 \mathrm{ff}$.

Schlegel, Rainer (2006): GKV-Wettbewerbsstärkungsgesetz und Selbstverwaltung aus sozialrechtlicher Sicht, Soziale Sicherheit, S. 378, 382.

Tennstedt, Florian/ Lohmann, Theodor: Gesetzgebungskunst im politischen Prozess, Wissenschaftliche Reihe der Otto-von-Bismarck-Stiftung, Manuskript S. 23. 